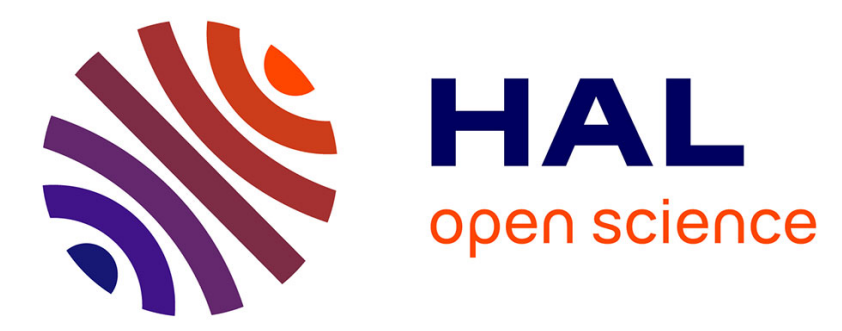

\title{
Low complexity robust adaptive beamformer based on parallel RLMS and Kalman RLMS
}

Ghattas Akkad, Ali Mansour, B.A. Elhassan, J. Srar, M. Najem, Frédéric Le Roy

\section{- To cite this version:}

Ghattas Akkad, Ali Mansour, B.A. Elhassan, J. Srar, M. Najem, et al.. Low complexity robust adaptive beamformer based on parallel RLMS and Kalman RLMS. 27th European Signal Processing Conference, EUSIPCO 2019, Sep 2019, A Coruna, Spain. 10.23919/EUSIPCO.2019.8902999 . hal02434614

\section{HAL Id: hal-02434614 \\ https://hal.science/hal-02434614}

Submitted on 21 Apr 2020

HAL is a multi-disciplinary open access archive for the deposit and dissemination of scientific research documents, whether they are published or not. The documents may come from teaching and research institutions in France or abroad, or from public or private research centers.
L'archive ouverte pluridisciplinaire HAL, est destinée au dépôt et à la diffusion de documents scientifiques de niveau recherche, publiés ou non, émanant des établissements d'enseignement et de recherche français ou étrangers, des laboratoires publics ou privés. 


\title{
Low Complexity Robust Adaptive Beamformer Based On Parallel RLMS and Kalman RLMS
}

\author{
Ghattas Akkad $^{1}$, Ali Mansour ${ }^{1}$, Bachar A. ElHassan ${ }^{2}$, Jalal Srar $^{3}$, Mohamad Najem $^{4}$, Frédéric Le Roy ${ }^{1}$ \\ ${ }^{1}$ Lab-STICC, UMR 6285, ENSTA Bretagne, Brest, France \\ ${ }^{2}$ Faculty of Engineering, Lebanese University, Tripoli, Lebanon \\ ${ }^{3}$ Electrical and Electronic Department, Misratah University, Misratah, Libya \\ ${ }^{4}$ CCE, Lebanese International University, Mount Lebanon, Lebanon \\ Ghattas.Akkad@ensta-bretagne.org
}

\begin{abstract}
To ease spectral congestion and enhance frequency reuse, researchers are targeting smart antenna systems using spatial multiplexing and adaptive signal processing techniques. Moreover, the accuracy and efficiency of such systems is highly dependent on the adaptive algorithms they employ. A popular, adaptive beamforming algorithm, widely used in smart antennas, is the Recursive Least Square (RLS) algorithm. While, the classical RLS implementation achieves high convergence, it still suffers from its inability to track the target of interest. Recently, a new adaptive algorithm called Recursive Least Square - Least Mean Square (RLMS) which employs a RLS stage followed by a Least Mean Square (LMS) algorithm stage and separated by an estimate of the array image vector, i.e. steering vector, has been proposed. RLMS outperforms previous RLS and LMS variants, with superior convergence and tracking capabilities, at the cost of a moderate increase in computational complexity. In this paper, an enhanced, low complexity parallel version of the cascade RLMS is presented by eliminating the need for computing the array image vector cascading stage. Hence, For an antenna of $N$ elements our strategy can reduce the complexity of the system by $20 N$ multiplications, $6 N$ additions and $2 N$ divisions. Moreover, a new Kalman based parallel RLMS (RKLMS) method is also proposed, where the LMS stage is replaced by a Kalman implementation of the classical LMS, and compared under low Signal to Interference plus Noise ratios (SINR). Simulation results show identical performance for the parallel RLMS, cascaded RLMS at 10dB and superior performance and robustness for the RKLMS on low SINR cases up to -10dB.
\end{abstract}

Index Terms-LMS, RLMS, Kalman Filter, Steering Vector, Multi Antenna, Adaptive Beamforming, KRLMS, MIMO, SINR, Spatial Multiplexing, RLS.

\section{INTRODUCTION}

The rapid increase of wireless systems (smartphones, internet connected devices, surveillance devices, etc.) created unparalleled challenges for researchers to overcome resulting problems, i.e. channel congestion, by enhancing spectral utilization [1], [2]. To resolve the underlying issues and reduce spectral congestion, smart antenna systems using spatial multiplexing and adaptive signal processing techniques have been employed. Such systems minimize the impact of interference by creating a steerable beam pattern, i.e. beamforming, for directional signal transmission and reception [3], [4]. Moreover, the performance of such systems in rapid and precise tracking is highly correlated with the performance and complexity of the operating beamforming algorithms [3], [4]. Numerous algorithms exist for realizing an adaptive beamforming systems with the most popular being the Least Mean Square (LMS) and Recursive Least Square (RLS). While the RLS algorithm can achieve faster convergence it does not offer acceptable tracking performance [5].

Several variants have been proposed for improving the convergence speed of LMS and tracking ability of RLS in time varying environments [4]. These techniques include variable step size LMS (VSSLMS) [6], the modified robust variable step size LMS (MRVSS) algorithm [7], the adaptive forgetting factor RLS algorithm (AFF-RLS) [8], the variable forgetting factor RLS (VFFRLS) and the extended recursive least square (EX-KRLS) algorithm [9].

In VSSLMS large step sizes are introduced at the beginning of the adaptation process and smaller ones as the algorithm approaches its steady state, allowing faster convergence and a lower residual error floor at the cost of a high increase in complexity [9]. To further increase VSSLMS noise immunity and tracking capabilities, the MRVSS algorithm has been proposed in [7]. Moreover, the robustness of the MRVSS and the improvement in the tracking ability of the RLS when implementing the VFFRLS, AFF-RLS and EX-KRLS algorithms are achieved at a considerably large increase in computation complexity [10], [11].

Recently, a novel RLMS approach for improving the convergence rate with superior tracking capabilities has been proposed [3], [4], [10], [11]. RLMS employs a RLS stage followed by a LMS stage separated by an estimate of the array image vector, i.e. steering vector with a delayed error feedback. This technique shows superior performance over previously discussed LMS and RLS variants with a relatively acceptable increase in computational complexity. Thus, for $N$ antenna elements, and in addition to the LMS stage, the complexity is increased by $20 \mathrm{~N}$ multiplications, $6 \mathrm{~N}$ additions and $2 N$ divisions for the steering vector estimate block.

In this context, the main motivation of this study is to further optimize the proposed RLMS in terms of complexity and robustness against low SINR, up to $-10 \mathrm{~dB}$ with an architecture suitable for a hardware based implementation. Such optimization is achieved by considering a parallel RLMS and parallel RLS with Kalman LMS (RKLMS) structures, eliminating the need for continuously computing the array image vector. 


\section{BACKGROUND REVIEW}

A brief background review on some of the well known and widely used adaptive algorithms for narrow-band complex signals is presented in this section. Let the input vector at the discrete time instant $k, \mathbf{x}(k)=\left[x_{1}(k), x_{2}(k), \ldots \ldots, x_{N}(k)\right]^{T}$, for a uniform linear antenna array with $N$ elements, be defined by

$$
\mathbf{x}(k)=\mathbf{a}_{\mathrm{d}} s_{\mathrm{d}}(k)+\mathbf{a}_{\mathrm{i}} s_{\mathrm{i}}(k)+\mathbf{n}(k)
$$

with $[.]^{T}$ being the matrix transpose, $s_{d}(k)$ and $s_{i}(k)$ are the desired and interfering signals, $\mathbf{a}_{\mathrm{d}}$ and $\mathbf{a}_{\mathrm{i}}$ are the $[N \times 1]$ complex array steering vectors for the desired signal and interference, and $\mathbf{n}(k)$ stands for the complex Additive White Gaussian Noise (AWGN) noise vector. by

A general form of the steering vectors $\mathbf{a}_{d}$ and $\mathbf{a}_{i}$ is given

$$
\mathbf{a}=\left[1, e^{-j 2 \pi \frac{D \sin (\theta)}{\lambda}}, \ldots, e^{-(N-1) j 2 \pi \frac{D \sin (\theta)}{\lambda}}\right]^{T}
$$

where the first antenna element acting as a reference, $\theta$ is the angle of arrival, $D$ is the distance between consecutive antenna elements, $\lambda$ is the signal wavelength. Thus, the output $y(k)$ of the beamformer subject to a linear combiner is given $b$

$$
y(k)=\mathbf{w}^{\mathbf{H}}(k) \mathbf{x}(k)
$$

where $[.]^{H}$ represents the matrix Hermitian transpose and $\mathbf{w}(k)$ is the array weight vector.

\section{A. Recursive Least Squares}

The Recursive Least Square (RLS) algorithm updates the arrays weight vector based on the minimization of a cost function. Which is defined by the sum of squared errors for a known sampling window [10] and is given by

$$
\zeta(k) \triangleq \sum_{i=1}^{k} \alpha^{k-1}\left|e_{R L S}(k-1)\right|^{2}
$$

where $e_{R L S}(k)=d(k)-y(k)$ is the error signal for the input and desired signals $\mathbf{x}(k)$ and $\mathbf{d}(k)$ respectively, $|$.$| denotes the$ modulus and $\alpha \epsilon] 0,1[$ is the exponential weighted forgetting factor. Hence, the weight vector update formula is as follow

$$
\begin{aligned}
& e_{R L S}(k)=d(k)-\mathbf{w}^{\mathbf{H}}(k-1) \mathbf{x}(k) \\
& \mathbf{L}(k)=\frac{\alpha^{-1} \mathbf{Q}^{-1}(k-1)}{1+\alpha^{-1} \mathbf{x}^{\mathbf{H}}(k) \mathbf{Q}^{-1}(k-1) \mathbf{x}(k)} \\
& \mathbf{w}(k)=\mathbf{w}(k-1)+\mathbf{L}(\mathrm{k}) \mathbf{x}(k) e^{*}(k)
\end{aligned}
$$

where $\mathbf{L}(k)$ is the gain matrix and the inverse signal autocorrelation matrix $\mathbf{Q}^{-1}(k)$ and the weight vector $\mathbf{w}(k-1)$ are given as follows

$$
\begin{aligned}
& \mathbf{Q}^{-1}(k)=\alpha^{-1} \mathbf{Q}^{-1}(k-1) \\
& -\frac{\alpha^{-2} \mathbf{Q}^{-1}(k-1) \mathbf{x}(k) \mathbf{x}^{\mathbf{H}}(k) \mathbf{Q}^{-1}(k-1)}{1+\alpha^{-1} \mathbf{x}^{\mathbf{H}}(k) \mathbf{Q}^{-1}(k-1) \mathbf{x}(k)}
\end{aligned}
$$

$$
\begin{aligned}
& \mathbf{w}(k-1)=\mathbf{Q}^{-1}(k-1) \mathbf{z}(k-1) \\
& \mathbf{z}(k)=\sum_{i=1}^{k} \alpha^{k-1} d^{*}(k) \mathbf{x}(k) \\
& \mathbf{Q}(k)=\sum_{i=1}^{k} \alpha^{k-1} \mathbf{x}(k) \mathbf{x}^{\mathbf{H}}(k)
\end{aligned}
$$

with $z(k)$ is the cross-correlation vector between the input and the desired signal and $\mathbf{Q}(k)$ is the input signal autocorrelation matrix [3]. While RLS offers fast convergence, it lacks acceptable tracking ability.

\section{B. Least Mean Squares}

The Least Mean Square (LMS) algorithm minimizes the mean square error (MSE) $e_{L M S}(k)$, between the input and desired signals $\mathbf{x}(k)$ and $\mathbf{d}(k)$, by using the steepest descent optimization method [9]. LMS is computationally efficient and is used to estimate the gradient of the error signal to update the weight vector of the antenna array. The weight vector $\mathbf{w}(k)$ is updated as follows

$$
\begin{aligned}
& e_{L M S}(k)=d(k)-y(k) \\
& \mathbf{w}(k+1)=\mathbf{w}(k)+\mu e_{L M S}^{*}(k) \mathbf{x}(k)
\end{aligned}
$$

where $\mu$ controls the magnitude of the gradient descent steps i.e. step size. While LMS offers superior tracking capabilities then RLS, it suffers from a trade off between its convergence speed and the steady state error [12]

\section{LMS Kalman Filter Representation}

Kalman's filter presents a realization of an optimal Bayesian estimator in a two step recursive process, i.e. prediction and correction. It is described by a state-space model which infers the parameters of interest from uncertain observations and is the core of many critical applications i.e. crowd-sensing [13], [14]. Therefore, Kalman's filter mathematical model for the LMS algorithm is given according to [12] by

$$
\begin{aligned}
& \mathbf{g}(k)=\frac{\mathbf{P}(k-1)}{\mathbf{x}^{\mathbf{H}}(k) \mathbf{P}(k-1) \mathbf{x}(k)+\sigma_{\mathrm{n}}^{2}} \mathbf{x}(k) \\
& \mathbf{w}(k)=\mathbf{w}(k-1)+\mathbf{g}(k)\left(\mathbf{d}(k)-\mathbf{x}^{\mathbf{H}}(k) \mathbf{w}(k-1)\right) \\
& \mathbf{P}(k)=\mathbf{P}(k-1)-\mathbf{g}(k) \mathbf{x}^{\mathbf{H}}(k) \mathbf{P}(k-1)
\end{aligned}
$$

where $\mathbf{g}(k)$ is the optimal learning gain vector, $\mathbf{P}(k-1)=$ $E\left[\widetilde{\mathbf{w}}(k-1) \widetilde{\mathbf{w}}^{\mathbf{H}}(k-1)\right]$ is the error co-variance matrix, $E[$. is the expectation operator, $\tilde{\mathbf{w}}(k-1) \triangleq \mathbf{w}_{o}(k-1)-\mathbf{w}(k-1)$ is the weight error vector, $\mathbf{w}_{o}(k)$ is the optimal weight vector and $\sigma_{n}^{2}=E\left[|\mathbf{n}(k)|^{2}\right]$ is the noise variance as given in [12].

\section{Cascade Form RLMS Beamformer}

The Recursive-Least mean square (RLMS) algorithm is a RLS stage followed by a LMS stage separated by an estimate of the array image vector with a delayed error feedback [4] as shown in figure 1. In RLMS, the output of the first stage $R L S_{1}, y_{R L S 1}(k)$, is multiplied by the estimate of the desired signal steering vector $\tilde{\mathbf{a}_{d}}$, forming the input, $\mathbf{x}_{2}(k)$, to the 


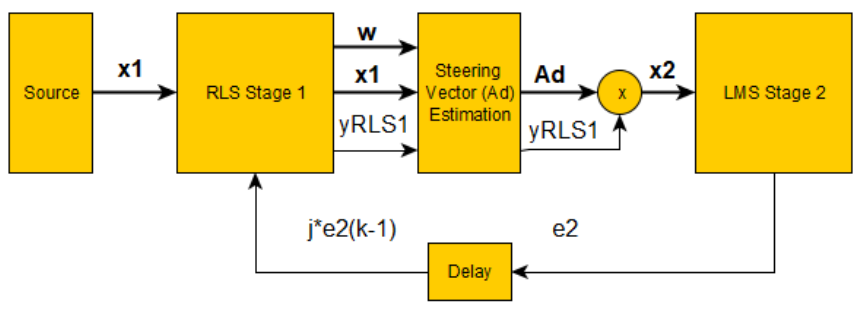

Fig. 1. Cascaded RLMS

second $L M S_{2}$ stage. Moreover, $\tilde{\mathbf{a}_{d}}$, is given by its stochastic approximation near convergence [15]

$$
\begin{aligned}
a_{d, m}(k) & \simeq \frac{E\left[w_{1, m}(k) x_{1, m}(k)\right]}{E\left[w_{1, m}\right] y_{R L S 1}(k)+\varepsilon} \\
& \simeq \frac{w_{1, m}(k) x_{1, m}(k)}{w_{1, m}(k) y_{R L S 1}(k)+\varepsilon}
\end{aligned}
$$

where $w_{1, m}(k), x_{1, m}(k)$ and $a_{d, m}$ are the $R L S_{1}$ tap weights, input signal and the estimate of the mth antenna element of the complex steering vector $\tilde{\mathbf{a}}_{d}$ at the time instant $k$ with $m \epsilon\{1,2,3 \ldots \ldots . N\}$. Where $\varepsilon$ is chosen as a small constant to prevent the division by zero. Moreover, a delayed version of the error signal $e_{2}(k)$ of the $L M S_{2}$ stage is fed-back to combine with that of the $R L S_{1}$ to form the overall error signal $e_{R L M S}(k)$ used to update the main tap weights of the $R L S_{1}$ stage. The overall error signal and the optimal tap weights are given by [10]

$$
\begin{aligned}
& e_{R L M S}(k)=e_{1}(k)-e_{2}(k-1) \\
& \mathbf{w}_{o}(k)=\mathbf{Q}^{-1}(k) \mathbf{z}_{R}(k)
\end{aligned}
$$

where $\mathbf{z}_{\mathbf{R}}(k)$ is an estimation of the input, $\mathbf{x}_{1}(k)$ assumed independent and identically distributed (iid), and the $L M S_{2}$ zero mean desired signal, $d_{1}(k)$, cross correlation vector

$$
\mathbf{z}_{R}(k)=E\left[d_{1}^{*}(k) \mathbf{x}_{1}(k)\right]
$$

while the RLMS presents accelerated convergence and superior tracking abilities, its cascaded form introduces high latency when targeting a hardware implementation with a division operation such as in equation (15). Furthermore, the system complexity is increased by $20 \mathrm{~N}$ multiplications, $6 \mathrm{~N}$ additions and $2 N$ divisions for the steering vector estimated in equation (15).

\section{PARALLEL RLMS}

As our main motivation is to propose an optimized parallel RLMS structure with reduced complexity and noise robustness. This section details the derivation of the RLMS basis for the RKLMS structure.

\section{A. Parallel RLMS}

As previously defined, the input to the $m^{\text {th }}$ antenna element for the $L M S$ stage, $x_{2, m}(k)$, is defined by

$$
x_{2, m}(k)=\frac{w_{1, m}(k) x_{1, m}(k)}{w_{1, m}(k) y_{R L S 1}(k)+\varepsilon} y_{R L S 1}(k)
$$

After a detailed inspection and since $\varepsilon$ is a negligible user introduced constant, equation (19) can be simplified to

$$
x_{2, m}(k) \approx x_{1, m}(k)
$$

thus the input to the $L M S$ stage, $\mathbf{x}_{2}(k)$, is none other then the reconstructed $R L S_{1}$ input $\mathbf{x}_{1}(k)$. The parallel RLMS can now be defined as shown in figure 2 .

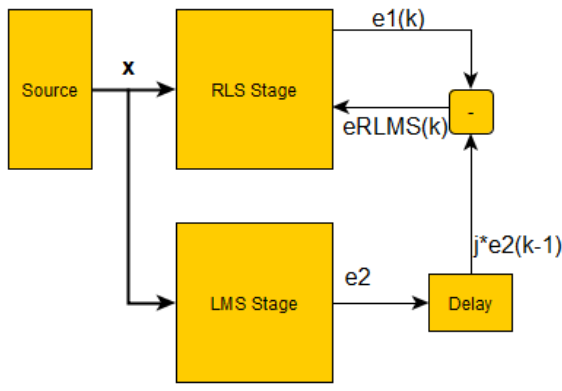

Fig. 2. Parallel RLMS

The parallel RLMS mean square error (MSE) is re-evaluated following the same assumptions in [10]: The propagation environment is time invariant, The components of the signal vector $\mathbf{x}(k)$ should be iid, and all signals are zero mean, wide sense stationary and spatially uncorrelated. Therefore, the MSE $\xi_{R L M S}$ is given by

$$
\begin{aligned}
& \zeta_{R L M S}(k) \triangleq \sum_{i=1}^{k} \alpha^{k-1} E\left[\left|e_{1}(k)-j e_{2}(k-1)\right|^{2}\right] \\
& =\sum_{i=1}^{k} \alpha^{k-1}\left\{E \left[e_{1}(k) e_{1}^{*}(k)+j e_{1}(k) e_{2}^{*}(k-1)\right.\right. \\
& \left.\left.-j e_{1}^{*}(k) e_{2}(k-1)+e_{2}(k-1) e_{2}^{*}(k-1)\right]\right\}
\end{aligned}
$$

where $j=\sqrt{-1}$ is the complex number resulting in a phase shift i.e. delay [9], $e_{1}(k)$ and $e_{2}(k-1)$ are the RLS and LMS errors respectively, $|$.$| signifies modulus and *$ is the complex conjugate operator. Moreover, the first term of equation (21) can be expressed as

$$
\begin{aligned}
& E\left[\left|e_{1}(k)\right|^{2}\right]=E\left[e_{1}(k) e_{1}^{*}(k)\right] \\
& =E\left[|d(k)|^{2}\right]-\mathbf{z}_{R}^{\mathbf{H}}(k) \mathbf{w}_{1}(k)-\mathbf{w}_{1}^{\mathbf{H}}(k) \mathbf{z}_{R}(k) \\
& +\mathbf{w}_{1}^{\mathbf{H}}(k) \mathbf{Q}(k) \mathbf{w}_{1}(k)
\end{aligned}
$$

correspondingly the last term of equation (21) can be expanded to become

$$
\begin{aligned}
& E\left[\left|e_{2}(k-1)\right|^{2}\right]=E\left[e_{2}(k-1) e_{2}^{*}(k-1)\right] \\
& =E\left[|d(k-1)|^{2}\right]-\mathbf{z}_{R}^{\mathbf{H}}(k-1) \mathbf{w}_{2}(k-1) \\
& -\mathbf{w}_{2}^{\mathbf{H}}(k-1) \mathbf{z}_{R}^{\mathbf{H}}(k-1)+\mathbf{w}_{2}^{\mathbf{H}}(k-1) \mathbf{Q}(k-1) \mathbf{w}_{2}(k-1)
\end{aligned}
$$

Furthermore, the second and third terms of equation (21) can be written as

$$
\begin{aligned}
& E\left[j e_{1}(k) e_{2}^{*}(k-1)\right]=j E\left[d(k) d^{*}(k-1)\right. \\
& -d(k) \mathbf{x}^{\mathbf{H}}(k-1) \mathbf{w}_{2}(k-1)-d^{*}(k-1) \mathbf{w}_{1}^{\mathbf{H}}(k) \mathbf{x}(k) \\
& \left.+\mathbf{w}_{1}^{\mathbf{H}}(k) \mathbf{x}(k) \mathbf{x}^{\mathbf{H}}(k-1) \mathbf{w}_{2}(k-1)\right]
\end{aligned}
$$


and

$$
\begin{aligned}
& E\left[-j e_{1}^{*}(k) e_{2}(k-1)\right]=-j E\left[d^{*}(k) d(k-1)\right. \\
& -d(k-1) \mathbf{x}^{\mathbf{H}}(k) \mathbf{w}_{1}(k)-d^{*}(k) \mathbf{w}_{2}^{\mathbf{H}}(k-1) \mathbf{x}(k-1) \\
& \left.+\mathbf{x}^{\mathbf{H}}(k) \mathbf{w}_{1}(k) \mathbf{w}_{2}^{\mathbf{H}}(k-1) \mathbf{x}(k-1)\right]
\end{aligned}
$$

where $\mathbf{w}_{2}(k-1)$ is the $L M S_{2}$ stage weight vector at time $k-1$. Thus, by using equations (22), (23), (24) and (25), the MSE defined in (21) can be rewritten as

$$
\begin{aligned}
& \zeta_{R L M S}(k)=\sum_{i=1}^{k} \alpha^{k-1}\left\{E\left[|d(k)|^{2}\right]-\mathbf{z}_{R}^{\mathbf{H}}(k) \mathbf{w}_{1}(k)\right. \\
& -\mathbf{w}_{1}^{\mathbf{H}}(k) \mathbf{z}_{R}^{\mathbf{H}}(k)+\mathbf{w}_{1}^{\mathbf{H}}(k) \mathbf{Q}(k) \mathbf{w}_{1}(k)+E\left[|d(k-1)|^{2}\right] \\
& -\mathbf{z}_{R}^{\mathbf{H}}(k-1) \mathbf{w}_{2}(k-1)-\mathbf{w}_{2}^{\mathbf{H}}(k-1) \mathbf{z}_{R}^{\mathbf{H}}(k-1) \\
& +\mathbf{w}_{2}^{\mathbf{H}}(k-1) \mathbf{Q}(k-1) \mathbf{w}_{2}(k-1)+j E\left[d(k) d^{*}(k-1)\right] \\
& -j E\left[d(k) \mathbf{x}^{\mathbf{H}}(k-1)\right] \mathbf{w}_{2}(k-1) \\
& -j \mathbf{w}_{1}^{\mathbf{H}}(k) E\left[d^{*}(k-1) \mathbf{x}(k)\right] \\
& +j E\left[d(k-1) \mathbf{x}^{\mathbf{H}}(k)\right] \mathbf{w}_{1}(k) \\
& -j E\left[d^{*}(k) d(k-1)\right]+j \mathbf{w}_{2}^{\mathbf{H}}(k-1) E\left[d^{*}(k) \mathbf{x}(k-1)\right] \\
& \left.-j E\left[\mathbf{x}^{\mathbf{H}}(k) \mathbf{w}_{1}(k) \mathbf{w}_{2}^{\mathbf{H}}(k-1) \mathbf{x}(k-1)\right]\right\}
\end{aligned}
$$

with $\mathbf{w}_{1}(k)$ being the tap weights of interest, the optimal weight vector, $\mathbf{w}_{o}(k)$, of $\mathbf{w}_{1}(k)$ can be obtained by differentiating equation (26) with respect to $\mathbf{w}^{\mathbf{H}_{1}}(k)$ using Wirtinger calculus [16], and equating the result to zero

$$
\begin{aligned}
& \frac{\partial \xi_{R L M S}(k)}{\partial \mathbf{w}_{1} \mathbf{H}_{1}(k)}=-\mathbf{z}_{R}^{\mathbf{H}}(k)+\mathbf{Q}(k) \mathbf{w}_{1}(k) \\
& -j E\left[d^{*}(k-1) \mathbf{x}(k)\right]+j E\left[\mathbf{x}(k) \mathbf{x}^{\mathbf{H}}(k-1)\right] \mathbf{w}_{2}(k-1)
\end{aligned}
$$

hence the optimal weight vector $\mathbf{w}_{o}(k)$ is represented as follows

$$
\begin{aligned}
\mathbf{w}_{o}(k) & =\mathbf{Q}^{-1}(k) \mathbf{z}_{R}^{\mathbf{H}}(k)+j \mathbf{Q}^{-1}(k) E\left[d^{*}(k-1) \mathbf{x}(k)\right] \\
& -j \mathbf{Q}^{-1}(k) E\left[\mathbf{x}(k) \mathbf{x}^{\mathbf{H}}(k-1)\right] \mathbf{w}_{2}(k-1)
\end{aligned}
$$

\section{Simulation Results}

We ran several Monte Carlo simulations with 200 realizations of 200 samples each with $N=8$ antenna elements. In addition, the input message and interfering signals are modeled as a random binary data stream modulated by binary phase shift keying (BPSK). The resulting signal is mixed with complex AWGN, with a signal to noise ratio $S N R=10 d B$. Simulations are conducted for LMS, RLS, KLMS, parallel RLMS, cascaded RLMS at $S N R=10 d B$. And using the parallel RLMS and proposed RKLMS for additional $S N R$ test case of $-10 d B$. The MSE is used to asses the convergence and performance of each case. In addition, simulation parameters for the LMS, RLMS variants and the Kalman LMS and RKLMS respectively, are given as, $\mu_{L M S}=0.05$, $\mu_{R L M S}=0.2, \alpha=0.98, \mathbf{P}(0)=0.5 I, \mathbf{Q}^{-1}(0)=0.025^{-1} I$, $\varepsilon=256 \times 10^{-12}$ and $\sigma_{n}^{2}=1$, where $I$ is a $[N \times N]$ identity matrix. Simulation results are in figures $3,4,5$ and 6 respectively.

The presented results in figure 3 show the identical performance of the cascade and parallel based RLMS stages and

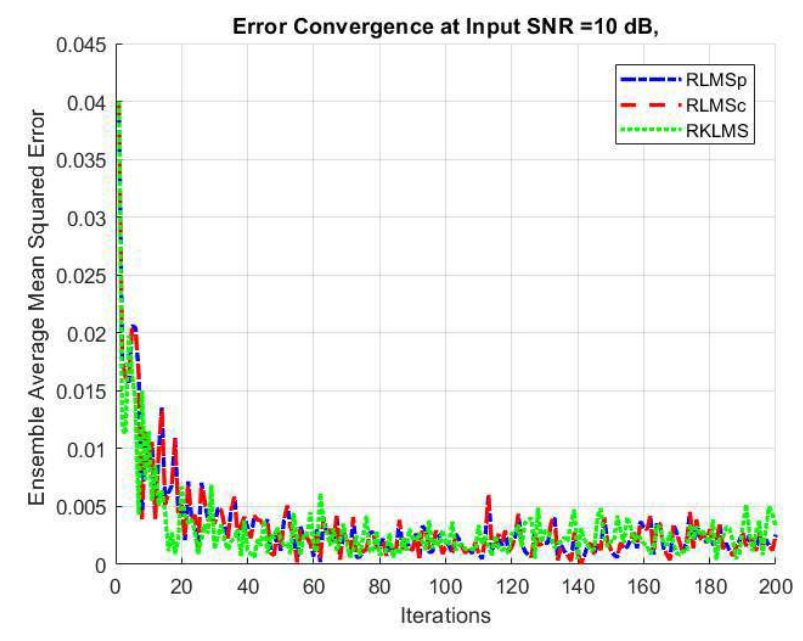

Fig. 3. RLMS and RKLMS Simulation Results for SNR 10dB

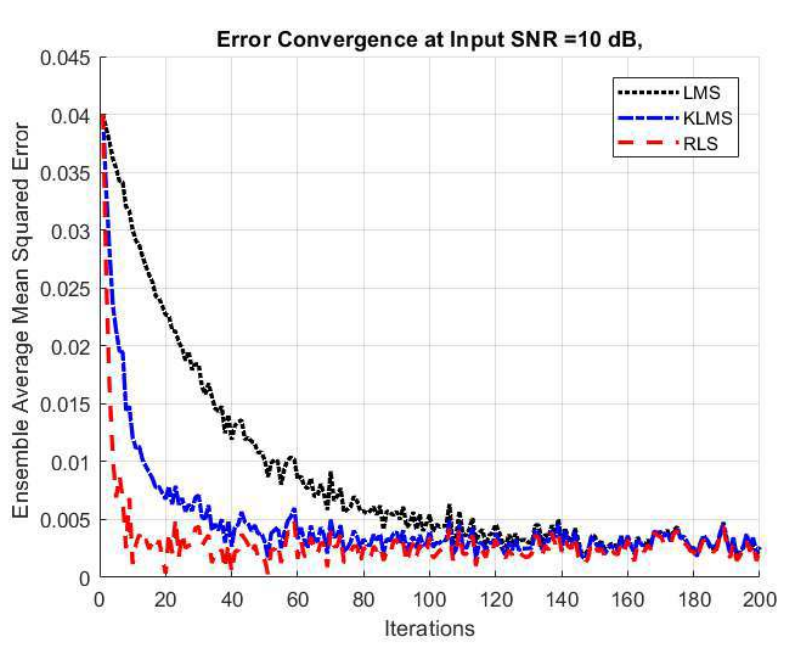

Fig. 4. LMS, KLMS and RLS, Simulation Results For SNR 10dB

better performance of the proposed RKLMS for $S N R=10 \mathrm{~dB}$. Moreover, the superior performance of the RKLMS to the traditional RLMS can be seen at $S N R=-10 d B$ from figures 5 and 6 respectively, where the classical RLMS diverged while the RKLMS converged. Furthermore, the following increase in performance is given at the cost of a small increase in complexity by replacing the classical LMS stage with the KLMS whose performance over the latter was demonstrated in figure 4. Thus,we can state that the parallel RLMS is indeed the cascade RLMS [4], [10] where $20 N$ multiplications, $6 N$ additions and $2 N$ divisions are reduced from the computational complexity. Thus, the resulting parallel structure has reduced the complexity and the latency. Therefore it becomes more suitable for a hardware implementation [17], [18].

\section{CONCLUSION}

In this paper, an efficient parallel RLMS adaptive beamformer, with identical performance over the traditional LLMS at $20 N$ multiplications, $6 N$ additions and $2 N$ divisions re- 


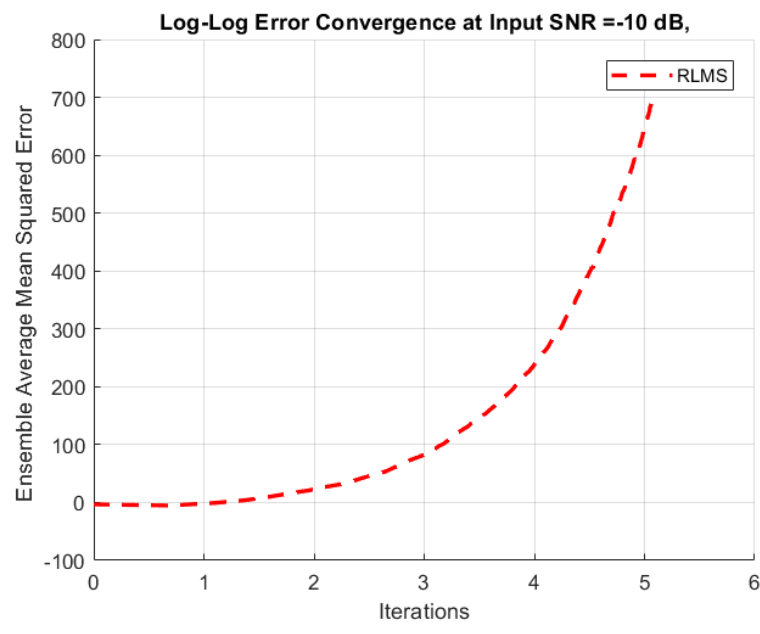

Fig. 5. RLMS Simulation Results For SNR -10dB Log-Log Plot

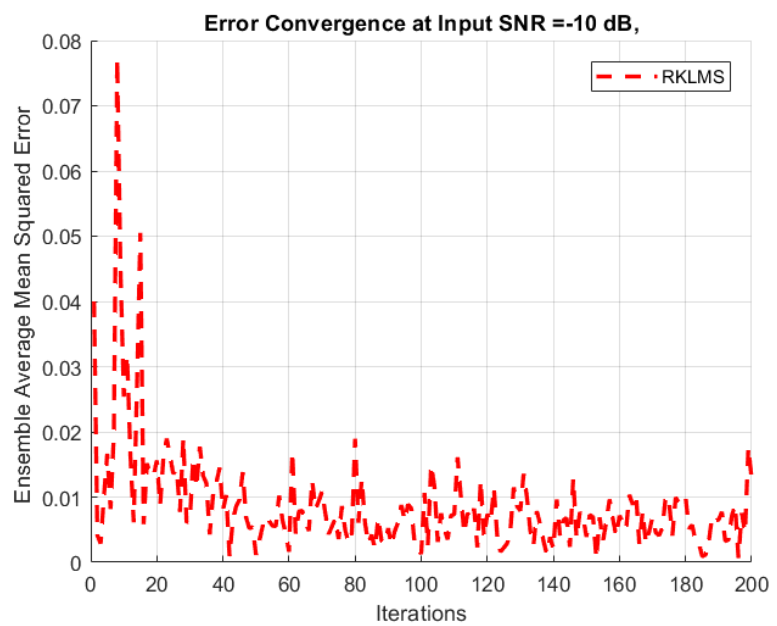

Fig. 6. RKLMS Simulation Results for SNR -10dB

duced complexity is presented. Moreover, a RKLMS is proposed for robustness against low SINR up to $S N R=-10 d B$ cases by modeling the classical LMS stage with a Kalman LMS filter. Experimental results have shown satisfactory convergence and identical performance of the parallel RLMS and cascade RLMS validating the proposed approach. The results also validated the robustness of the RKLMS in low SINR scenarios with superior performance over the previously stated algorithm at a slight increase in complexity. While, presented simulations show promising results, they only consider the preset simulation parameters. However, the RLMS and RKLMS performance may be further optimized for additional robustness by wisely selecting the preset value of the initial parameters, i.e. step size and the error co-variance matrix. Thus, future work includes performing stability analysis to deduce the optimal initial parameters and implementing the parallel RKLMS structure on re-configurable hardware such as FPGA.

\section{REFERENCES}

[1] T. S. RAPpaport, S. Sun, R. MAYzus, H. ZhaO, Y. Azar, K. WANG, G. N. Wong, J. K. SChulZ, M. SAmimi, AND F. GUTIERREZ, "MILLIMETER WAVE MOBILE COMMUNICATIONS FOR 5G CELLULAR: IT WILL WORK!," IEEE Access, VOL. 1, PP. 335-349, 2013.

[2] A. Mansour, R. Mesleh, and M. Abaza, "NeW Challenges IN WIRELESS AND FREE SPACE OPTICAL COMMUNICATIONS," Optics and Lasers in Engineering, VOL. 89, PP. 95 - 108, 2017.

[3] J. A. SRAR AND K. ChUnG, "PERFORMANCE OF RLMS ALgOrithm IN ADAPTIVE ARRAY BEAM FORMING," IN 2008 11th IEEE Singapore International Conference on Communication Systems, PP. 493-498, Nov 2008

[4] J. A. SRAR AND K. Chung, "Rlms Algorithm FOR FIXED OR ADAPTIVE BEAMFORMING," IN 2009 IEEE 9th Malaysia International Conference on Communications (MICC), PP. 163-167, DEC 2009.

[5] F.-B. Ueng, J.-D. Chen, and S.-H. Cheng, "SMart antennas FOR MULTIUSER DS/CDMA COMMUNICATIONS IN MULTIPATH FADING CHANNELS," IN Eighth IEEE International Symposium on Spread Spectrum Techniques and Applications - Programme and Book of Abstracts (IEEE Cat. No.04TH8738), PP. 400-404, AUG 2004.

[6] Z. XIUBING et al., "A NEW MODIFIED ROBUST VARIABLE STEP SIZE LMS ALGORITHM," IN Industrial Electronics and Applications, 2009. ICIEA, PP. 2699-2703, IEEE, 2009.

[7] E. M. Lobato, O. J. Tobias, And R. SEARA, "Stochastic ModELING OF THE TRANSFORM-DOMAIN Elms ALGORITHM," IEEE Transactions on Signal Processing, VOL. 56, PP. 1840-1852, MAY 2008

[8] C. Paleologu, J. Benesty, and S. Ciochina, "A robust VariABLE FORGETTING FACTOR RECURSIVE LEAST-SQUARES ALGORITHM FOR SYSTEM IDENTIFICATION," IEEE Signal Processing Letters, VOL. 15, PP. 597-600, 2008.

[9] J. A. Srar, K. Chung, and A. Mansour, "Adaptive array BEAMFORMING USING A COMBINED LMS-LMS ALGORITHM," IEEE Transactions on Antennas and Propagation, VOL. 58, PP. 3545-3557, Nov 2010

[10] J. A. SRAR AND K. Chung, "Adaptive ARray BeAm Forming USING A COMBINED RLS-LMS ALGORITHM," IN 2008 14th Asia-Pacific Conference on Communications, PP. 1-5, OCT 2008.

[11] J. A. SRAR AND K. Chung, "AdAPtive RLMS Algorithm FOR ANTENNA ARRAY BEAMFORMING," IN TENCON, PP. 1-6, JAN 2009.

[12] D. P. Mandic, S. Kanna, and A. G. Constantinides, "On THE INTRINSIC RELATIONSHIP BETWEEN THE LEAST MEAN SQUARE AND Kalman Filters [Lecture nOtes]," IEEE Signal Processing Magazine, VOL. 32, PP. 117-122, Nov 2015.

[13] S. SARKKa, A. Solin, and J. Hartikainen, "Spatiotemporal LEARNING VIA INFINITE-DIMENSIONAL BAYESIAN FILTERING AND SMOOTHING: A LOOK AT GAUSSIAN PROCESS REGRESSION THROUGH KAlman FILTERING," IEEE Signal Processing Magazine, Vol. 30, PP. 51-61, JULY 2013.

[14] D. DARDARI, G. PASOlini, AND F. ZABINI, "AN EFFICIENT METHOD FOR PHYSICAL FIELDS MAPPING THROUGH CROWDSENSING," Pervasive and Mobile Computing, VOL. 48, PP. 69 - 83, 2018.

[15] G. AkKad, A. Mansour, B. Elhassan, J. Srar, M. Najem, AND F. LEROY, "AN EFFICIENT NON-BLIND STEERING VECTOR ESTIMATION TECHNIQUE FOR ROBUST ADAPTIVE BEAMFORMING WITH MULTISTAGE ERROR FEEDBACK," IN Intelligent Decision Technologies 2019 (I. CZArnowski, R. J. Howlett, And L. C. JAin, EDS.), (SINGAPORE), PP. 13-23, SPRINGER SINGAPORE, 2019.

[16] P. BOUBOULIS AND S. THEODORIDIS, "EXTENSION OF WIRTINGER'S CALCULUS TO REPRODUCING KERNEL HILBERT SPACES AND THE COMPLEX KERNEL LMS," IEEE Transactions on Signal Processing, VOL. 59, PP. 964-978, MARCH 2011

[17] G. AkKad, A. Mansour, B. ElHassan, F. L. Roy, and M. NAJEM, "TWIDDLE FACTOR GENERATION USING CHEBYSHEV POLYNOMIALS AND HDL FOR FREQUENCY DOMAIN BEAMFORMING," IN $A p$ plications in Electronics Pervading Industry, Environment and Society, Springer Lecture Notes in Electrical Engineering, SPRINGER, SEPT. 2018.

[18] G. Akkad, A. Mansour, B. Elhassan, F. L. Roy, and M. NaJEM, "FFT RADIX-2 AND RADIX-4 FPGA ACCELERATION TECHNIQUES USING HLS AND HDL FOR DIGITAL COMMUNICATION SYSTEMS," IN 2018 IEEE International Multidisciplinary Conference on Engineering Technology (IMCET), PP. 1-5, NOV 2018. 